\title{
Pseudocirrhosis caused by regorafenib in an advanced rectal cancer patient with multiple liver metastases
}

\author{
KENSUKE KUMAMOTO $^{1}$, SHUNGO ENDO ${ }^{1}$, NORIYUKI ISOHATA ${ }^{1}$, AZUMA NIREI ${ }^{2}$, \\ DAIKI NEMOTO $^{1}$, KENICHI UTANO ${ }^{1}$, TAKURO SAITO ${ }^{2}$ and KAZUTOMO TOGASHI ${ }^{1}$ \\ Departments of ${ }^{1}$ Coloproctology and ${ }^{2}$ Surgery, Aizu Medical Center, \\ Fukushima Medical University, Aizuwakamatsu, Fukushima 969-3492, Japan
}

Received May 13, 2016; Accepted November 18, 2016

DOI: $10.3892 / \mathrm{mco} .2016 .1096$

\begin{abstract}
A 70-year-old man who was diagnosed with unresectable advanced rectal cancer with multiple liver metastases, received oxaliplatin-based treatment with bevacizumab as first-line chemotherapy and irinotecan-based treatment with bevacizumab as second-line chemotherapy for a total of 17 months. The patient was treated with regorafenib (160 mg/day for 3 weeks) as third-line chemotherapy. Following completion of one course of regorafenib treatment, the patient complained of abdominal distension. Computed tomography (CT) examination identified liver atrophy and massive ascites, while no such symptoms were observed prior to the regorafenib treatment. Blood testing revealed increases in the aspartate aminotransferase (AST), alanine aminotransferase (ALT) and alkaline phosphatase (ALP) levels. The patient was admitted to the Aizu Medical Center (Aizuwakamatsu, Japan). Approximately 2,000 ml of ascitic fluid were aspirated daily for 1 week by abdominal puncture. The patient was administered oral diuretics, including $20 \mathrm{mg}$ /day of furosemide and $25 \mathrm{mg} /$ day of spironolactone. Albumin was administered to correct the albumin deficit. The levels of AST, ALT and ALP were decreased from the peak value reported on admission and the patient was discharged from our hospital 16 days following treatment initiation. The CT examination after 1 month revealed that the volume of the liver had been restored and the ascites had disappeared. Furthermore, almost all the liver metastases were reduced in size. The carcinoembryonic antigen level, which was elevated prior to regorafenib treatment, also decreased to normal.
\end{abstract}

Correspondence to: Dr Kensuke Kumamoto, Department of Coloproctology, Aizu Medical Center, Fukushima Medical University, 21-2 Tanisawa, Kawahigashi, Aizuwakamatsu, Fukushima 969-3492, Japan

E-mail:kumamotk@fmu.ac.jp

Key words: regorafenib, hepatotoxicity, pseudocirrhosis, chemotherapy

\section{Introduction}

Chemotherapy with molecular-targeted drugs improves the survival rate of patients with unresectable advanced colorectal cancer (CRC). Although chemotherapy is effective for a subset of patients with CRC, the incidence of adverse events (AEs) often affects treatment continuity. Regorafenib (1), which is an oral multikinase inhibitor, is commonly used as third- or fourth-line chemotherapy in refractory CRC patients (2). Several clinical trials (2-4) have demonstrated the efficacy of regorafenib for refractory CRC patients. The most frequent regorafenib-related AEs of grade $\geq 3$ in the CORRECT trial were hand-foot skin reaction, fatigue, diarrhea, hypertension and rash or desquamation (2). Regarding the incidence of regorafenib-related hepatotoxicity, including hyperbilirubinaemia and elevation of aspartate aminotransferase (AST) and alanine aminotransferase (ALT) levels, it was relatively low in a non-Japanese population, while it was high in a Japanese population (5). These results were supported with the CONCUR trial (3), which was performed by institutions in Asia.

The present study reports the case of a CRC patient with multiple liver metastases, who developed liver atrophy and massive ascites following regorafenib treatment. To the best of our knowledge, this is the first report of a rare AE induced by regorafenib treatment.

\section{Case report}

A 70-year-old man, who was diagnosed with advanced rectal cancer with multiple liver metastases (Fig. 1A), received chemotherapy withoutsurgical treatment from November,2013 onwards. First, the patient was treated with XELOX + bevacizumab (2,000 $\mathrm{mg}$ /day capecitabine on days $1-14$ and $150 \mathrm{mg}$ oxaliplatin on day $1+150 \mathrm{mg}$ bevacizumab on day 1) for three cycles. The regimen was changed to SOX + bevacizumab (100 mg/day S-1 on days $1-14,150 \mathrm{mg}$ oxaliplatin on day $1+150 \mathrm{mg}$ bevacizumab on day 1) due to severe AEs, including hand-foot syndrome. Ten cycles of the SOX treatment were administered over 10 months, achieving stable disease, until the carcinoembryonic antigen (CEA) level was elevated to $8.5 \mathrm{ng} / \mathrm{ml}$ (normal range, $<5.0 \mathrm{ng} / \mathrm{ml}$ ) (Fig. 2). IRIS + bevacizumab (100 mg/day S-1 on days $1-14,150 \mathrm{mg}$ irinotecan on day $1+150 \mathrm{mg}$ bevacizumab on day 1) treatment was subsequently administered. However, the 

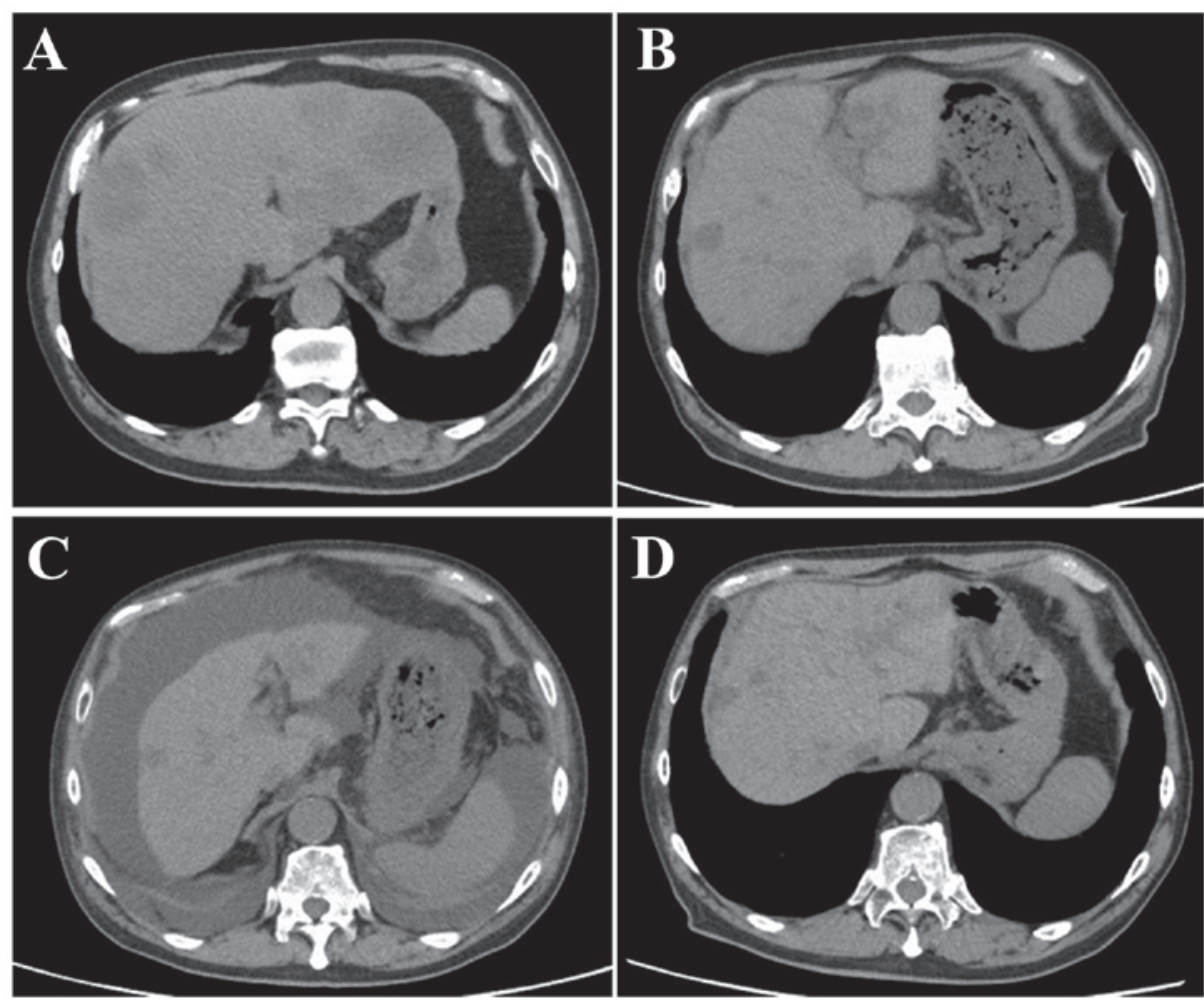

Figure 1. Abdominal computed tomography (A) at the time of initial diagnosis; (B) prior to administration of regorafenib; (C) following one cycle of regorafenib administration; and (D) 1 month after discharge.

IRIS + bevacizumab treatment was not effective for the patient, reflected by the increase in the CEA level to $13.2 \mathrm{ng} / \mathrm{ml}$ (Fig. 2). The primary rectal cancer almost disappeared during chemotherapy. The RAS status could not be examined. Regorafenib (160 mg/day for 3 weeks) was then administered as third-line chemotherapy. Following completion of one course, the patient complained of abdominal distension. Although no abnormal findings were observed on computed tomography (CT) examination prior to the regorafenib treatment (Fig. 1B), liver atrophy and massive ascites were identified (Fig. 1C). The blood tests after regorafenib treatment revealed elevated levels of AST, ALT and alkaline phosphatase (ALP) to 55 IU/1 (normal range, 12-32 IU/1), $40 \mathrm{IU} / 1$ (normal range, 6-30 IU/1) and 505 IU/1 (normal range, 109-335 IU/1), respectively (Table I). For the evaluation of liver function, ${ }^{99 \mathrm{~m}} \mathrm{Tc}$-galactosyl human serum albumin scintigraphy, which reflects the number and function of hepatocytes, was performed. The uptake ratio of the liver to the liver plus heart at 15 min was 0.77 . The estimated value of indocyanine green clearance was $21 \%$. Endoscopic examination of the esophagus revealed mild esophageal varices without red color sign. The patient was admitted to the Aizu Medical Center (Aizuwakamatsu, Japan) and 2,000 ml of ascitic fluid were aspirated daily for 1 week by abdominal puncture. The patient was administered oral diuretics, including $20 \mathrm{mg} /$ day of furosemide and $25 \mathrm{mg} /$ day of spironolactone. Albumin was administered to correct the albumin deficit. The levels of AST, ALT and ALP decreased from the peak values observed on admission (Fig. 3). The patient was discharged from the hospital 16 days after the treatment. The CT examination after 1 month revealed that the volume of the liver had recovered and the ascites had disappeared (Fig. 1D). Furthermore, almost all the

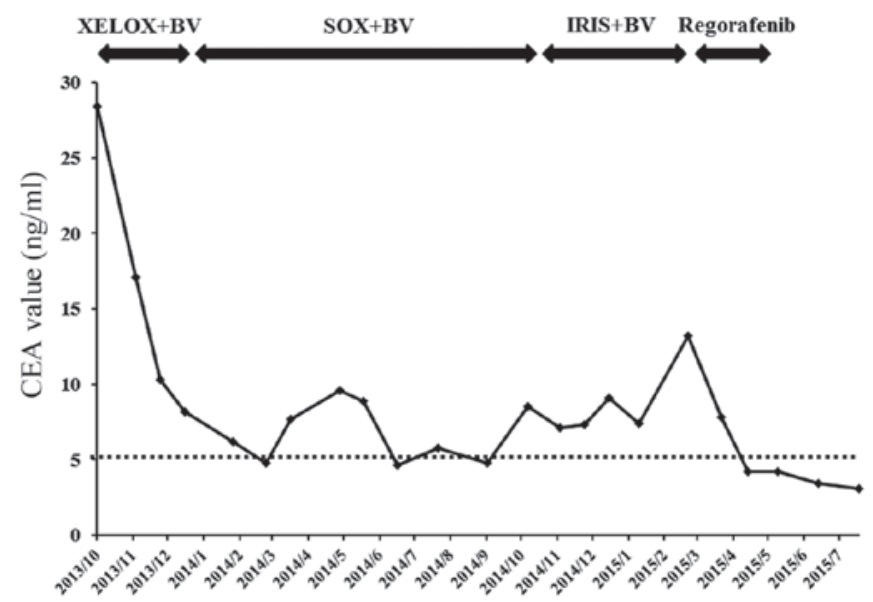

Figure 2. Time course of the changes in the CEA level with different chemotherapeutic regimens. CEA, carcinoembryonic antigen; BV, bevacizumab.

liver metastases were reduced in size. The CEA level, which was elevated prior to regorafenib treatment, was reduced to normal (Fig. 2).

\section{Discussion}

The present study reported the case of a CRC patient with liver atrophy and massive ascites induced by regorafenib treatment. The patient had received oxaliplatin-based and irinotecan-based chemotherapy prior to regorafenib. Although accumulation of these chemotherapeutic agents may be associated with liver dysfunction, liver atrophy was observed following administration 
Table I. Blood test results following regorafenib treatment.

\begin{tabular}{|c|c|}
\hline Parameters & Values \\
\hline WBC count & $4,320 / \mu 1$ \\
\hline RBC count & $4.01 \times 10^{4} / \mu 1$ \\
\hline Hemoglobin & $12.4 \mathrm{~g} / \mathrm{dl}$ \\
\hline Hematocrit & $39.4 \%$ \\
\hline PLT count & $6.2 \times 10^{3} / \mathrm{ml}$ \\
\hline Total protein & $5.0 \mathrm{~g} / \mathrm{dl}$ \\
\hline Albumin & $1.8 \mathrm{~g} / \mathrm{dl}$ \\
\hline $\mathrm{AST}$ & $55 \mathrm{IU} / 1$ \\
\hline ALT & $40 \mathrm{IU} / 1$ \\
\hline $\mathrm{LDH}$ & $328 \mathrm{IU} / 1$ \\
\hline ALP & $505 \mathrm{IU} / 1$ \\
\hline$\gamma$-GTP & $80 \mathrm{IU} / 1$ \\
\hline Total bilirubin & $0.6 \mathrm{mg} / \mathrm{dl}$ \\
\hline BUN & $27.4 \mathrm{mg} / \mathrm{dl}$ \\
\hline Creatinine & $1.29 \mathrm{mg} / \mathrm{dl}$ \\
\hline $\mathrm{Na}$ & 134 mEq/l \\
\hline $\mathrm{K}$ & $4.7 \mathrm{mEq} / 1$ \\
\hline $\mathrm{Cl}$ & 106 mEq/1 \\
\hline HPT & $91.6 \%$ \\
\hline PT & $100 \%$ \\
\hline PTINR & 1.0 \\
\hline APTT & $31.5 \mathrm{sec}$ \\
\hline Fibrinogen & $209.3 \mathrm{mg} / \mathrm{dl}$ \\
\hline ATIII & $69 \%$ \\
\hline FDP & $10.9 \mu \mathrm{g} / \mathrm{ml}$ \\
\hline D-dimer & $5.8 \mu \mathrm{g} / \mathrm{ml}$ \\
\hline Ammonia & $65 \mu \mathrm{g} / \mathrm{dl}$ \\
\hline
\end{tabular}

WBC, white blood cell; RBC, red blood cell; PLT, platelet; AST, aspartate aminotransferase; ALT, alanine aminotransferase; LDH, lactate dehydrogenase; ALP, alkaline phosphatase; $\gamma$-GTP, $\gamma$-glutamyl transpeptidase; BUN, blood urea nitrogen; HPT, heparin protamine titration; PT, prothrombin time; PTINR, prothrombin time/international normalized ratio; APTT, activated partial thromboplastin time; ATIII, antithrombin III; FDP, fibrin degradation products.

of regorafenib alone and the size of the liver was restored following discontinuation of regorafenib treatment. Therefore, regorafenib was considered to be the cause of liver atrophy and massive ascites in the present case. To the best of our knowledge, this is the first report of atrophic changes of the liver and massive ascites caused by regorafenib treatment in a CRC patient with multiple liver metastases. Recently, chemotherapeutic agents, including molecular-targeted drugs, antivascular endothelial growth factor antibodies, anti-epidermal growth factor receptor antibodies and multikinase inhibitors, have been administered to refractory CRC patients and have improved their survival rate. However, careful observation for AEs is required during chemotherapeutic treatment. The most frequent regorafenibrelated AEs of grade $\geq 3$ in the CORRECT trial were hand-foot skin reaction, fatigue, diarrhea, hypertension and rash or desquamation (2). Drug-induced hepatotoxicity is a common

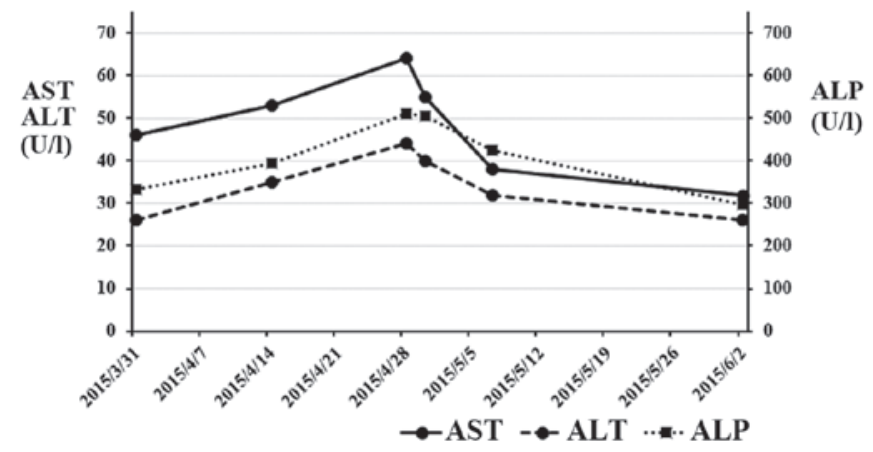

Figure 3. Changes in the AST, ALT and ALP levels during regorafenib treatment. AST, aspartate aminotransferase; ALT, alanine aminotransferase; ALP, alkaline phosphatase.

AE, although the frequency and the type of drug-induced liver damage differ depending on the drugs administered. Regarding hepatotoxicity caused by regorafenib, the incidence of all-grade hepatotoxicity, including hyperbilirubinaemia (8\%), AST elevation $(2 \%)$ and ALT elevation $(<1 \%)$, was relatively low in a non-Japanese population, while the incidence of hyperbilirubinaemia (15\%), AST elevation (19\%) and ALT elevation (12\%) was high in a Japanese population (5). These results were supported by the CONCUR trial (3), a clinical trial on non-Japanese Asian patients. The incidence of hyperbilirubinemia, AST elevation and ALT elevation was $48.5,31.6$ and $31.6 \%$, respectively. In the present case, the blood tests associated with liver function revealed an increase and a peak at 4 weeks after the initiation of regorafenib treatment, which corresponded to grade 1 hepatotoxicity, according to the Common Terminology Criteria for Adverse Events, version 3.0 (https://ctep.cancer.gov/protocolDevelopment/ electronic_applications/docs/ctcaev3.pdf). Other AEs, including hand-foot skin reaction, fatigue, diarrhea, hypertension and rash or desquamation, were not observed at that time. Therefore, it would be difficult to detect liver damage only from the results of the blood tests if the patient had not complained of abdominal distension.

In general, drug-induced liver injury (DILI) is classified into intrinsic and idiosyncratic liver injury. The former is induced by drug toxicity in a dose-dependent manner, whereas the latter is further divided into allergic and metabolic idiosyncratic liver injuries, which are associated with host factors, including immune response and metabolism associated with genetic polymorphisms. The majority of DILIs are idiosyncratic. The area under the curve of regorafenib treatment varied for intake of the same dose, depending on the individual. Regorafenib was metabolized to M-2 and M-5, which act through CYP3A4 (6). UGT1A9 is associated with the inactivation of these products through glucuronate conjugation (6). Therefore, the UGT1A9 gene polymorphism may be associated with the toxicity of regorafenib. Sorafenib is also a multikinase inhibitor, which has a similar structure to regorafenib (7). Sorafenib is also metabolized by CYP3A4-mediated oxidation and UGT1A9-mediated glucuronidation. Ye et al (8) reported that sorafenib metabolism was significantly altered in the liver tumor tissue of a hepatocellular carcinoma patient, due to an evident decrease of the expression level of CYP3A4 and UGT1A9. Boudou-Rouquette et al (9) reported that the UGT1A9 polymorphism (rs17868320) was 
significantly associated with grade $>2$ diarrhea in patients treated with sorafenib. Although there were no data confirming that the patient had the UGT1A9 polymorphism, it was hypothesized that these genetic polymorphisms confer a clinical benefit but induce severe toxicity in the patient.

Similar changes on imaging were previously reported in breast cancer with multiple liver metastases $(10,11)$ and other cancers with multiple liver metastases, including pancreatic (12), esophageal (13) and thyroid cancer (14). The morphological changes of the liver in these cases are referred to as 'pseudocirrhosis'. Pseudocirrhosis is a radiological term that indicates a shape similar to that of macronodular cirrhosis in the absence of the typical histopathological findings of cirrhosis, and it occurs in cancer patients with multiple liver metastases during chemotherapy. The progression and regression of liver metastases may cause pseudocirrhosis. Although the precise mechanism underlying the development of pseudocirrhosis remains unknown, the response to systemic chemotherapeutic agents may induce cirrhotic changes with tumor shrinkage following chemotherapy. Previous reports (10-12) have indicated that pseudocirrhosis may be associated with nodular regenerative hyperplasia (NRH) caused by chemotherapy-induced hepatic injury. NRH is characterized by widespread transformation of normal liver parenchyma into hyperplastic regenerative nodules without bridging fibrosis, a characteristic that distinguishes this entity from liver cirrhosis (15). This injury is characteristically asymptomatic in its early phases, with only mild elevations in transaminase levels (16). Oxaliplatin is a well-known causative drug for the development of NRH (17). Previous studies on breast cancer reported that multiple liver metastases worsened when atrophic changes occurred in the liver $(10,11)$. Kang et al (12) reported a reversible change in a pancreatic cancer patient with multiple liver metastases, who received 8 cycles of chemotherapy with gemcitabine and 5-fluorouracil. When liver atrophy was observed during treatment, the values of the liver enzymes were elevated, while the level of carbohydrate antigen 19-9 was markedly decreased. This was also the case in our patient, although the chemotherapeutic agents were different. In the present case, the volume of the liver was markedly decreased, with a mild elevation of the aminotransferase levels. Following withdrawal of regorafenib, the volume of the liver was almost completely restored to that prior to the initiation of regorafenib treatment. Furthermore, the CEA level returned to normal following treatment with regorafenib. The majority of cancer patients with pseudocirrhosis have a poor prognosis due to progression of liver metastases. However, effective treatment may also induce cirrhotic changes due to the potent antitumor effect.

Symptomatic treatment was administered for the liver dysfunction, in addition to withdrawal of regorafenib, according to the treatment for cirrhosis with ascites. In the present case, the patient survived after severe regorafenib-induced hepatic injury. The course of treatment in the present as well as another case (12) suggests that pseudocirrhosis with a mild elevation of aminotransferase levels during chemotherapy may be reversed by discontinuation of the chemotherapeutic agents. In such cases, where the chemotherapeutic agents administered are potent enough to induce liver injury and reversible pseudocirrhosis, potent antitumor effects may also be expected. This study presents an extremely rare case of recovery from pseudocirrhosis; however, the majority of cancer patients with pseudocirrhosis induced by chemotherapeutic agents eventually succumb due to cancer progression. Clinicians should be aware that regorafenib may induce atrophic changes of the liver and generate massive ascites in CRC patients with multiple liver metastases.

\section{References}

1. Wilhelm SM, Dumas J, Adnane L, Lynch M, Carter CA, Schütz G, Thierauch KH and Zopf D: Regorafenib (BAY 73-4506): A new oral multikinase inhibitor of angiogenic, stromal and oncogenic receptor tyrosine kinases with potent preclinical antitumor activity. Int J Cancer 129: 245-255, 2011.

2. Grothey A, Van Cutsem E, Sobrero A, Siena S, Falcone A, Ychou M, Humblet Y, Bouché O, Mineur L, Barone C, et al; CORRECT Study Group: Regorafenib monotherapy for previously treated metastatic colorectal cancer (CORRECT): An international, multicentre, randomised, placebo-controlled, phase 3 trial. Lancet 381: 303-312, 2013.

3. Li J, Qin S, Xu R, Yau TC, Ma B, Pan H, Xu J, Bai Y, Chi Y, Wang L, et al; CONCUR Investigators: Regorafenib plus best supportive care versus placebo plus best supportive care in Asian patients with previously treated metastatic colorectal cancer (CONCUR): A randomised, double-blind, placebo-controlled, phase 3 trial. Lancet Oncol 16: 619-629, 2015.

4. Kim ST, Kim TW, Kim KP, Kim TY, Han SW, Lee JY, Lim SH, Lee MY, Kim H and Park YS: Regorafenib as Salvage Treatment in Korean Patients with Refractory Metastatic Colorectal Cancer. Cancer Res Treat 47: 790-795, 2015.

5. Yoshino T, Komatsu Y, Yamada Y, Yamazaki K, Tsuji A, Ura T, Grothey A, Van Cutsem E, Wagner A, Cihon F, et al: Randomized phase III trial of regorafenib in metastatic colorectal cancer: Analysis of the CORRECT Japanese and non-Japanese subpopulations. Invest New Drugs 33: 740-750, 2015.

6. Rey JB, Launay-Vacher V and Tournigand C: Regorafenib as a single-agent in the treatment of patients with gastrointestinal tumors: An overview for pharmacists. Target Oncol 10: 199-213, 2015.

7. Carr BI, Cavallini A, Lippolis C, D'Alessandro R, Messa C, Refolo MG and Tafaro A: Fluoro-Sorafenib (Regorafenib) effects on hepatoma cells: Growth inhibition, quiescence, and recovery. J Cell Physiol 228: 292-297, 2013.

8. Ye L, Yang X, Guo E, Chen W, Lu L, Wang Y, Peng X, Yan T, Zhou F and Liu Z: Sorafenib metabolism is significantly altered in the liver tumor tissue of hepatocellular carcinoma patient. PLoS One 9: e96664, 2014.

9. Boudou-RouquetteP,NarjozC,GolmardJL,Thomas-SchoemannA, Mir O, Taieb F, Durand JP, Coriat R, Dauphin A, Vidal M, et al: Early sorafenib-induced toxicity is associated with drug exposure and UGTIA9 genetic polymorphism in patients with solid tumors: A preliminary study. PLoS One 7: e42875, 2012.

10. Lee SL, Chang ED, Na SJ, Kim JS, An HJ, Ko YH and Won HS: Pseudocirrhosis of breast cancer metastases to the liver treated by chemotherapy. Cancer Res Treat 46: 98-103, 2014.

11. Jeong WK, Choi SY and Kim J: Pseudocirrhosis as a complication after chemotherapy for hepatic metastasis from breast cancer. Clin Mol Hepatol 19: 190-194, 2013.

12. Kang SP, Taddei T, McLennan B and Lacy J: Pseudocirrhosis in a pancreatic cancer patient with liver metastases: A case report of complete resolution of pseudocirrhosis with an early recognition and management. World J Gastroenterol 14: 1622-1624, 2008.

13. Kobashigawa C, Nakamoto M, Hokama A, Hirata T, Kinjo F and Fujita J: Pseudocirrhosis in metastatic esophageal cancer. South Med J 103: 488-489, 2010.

14. Harry BL, Smith ML, Burton JR Jr, Dasari A, Eckhardt SG and Diamond JR: Medullary thyroid cancer and pseudocirrhosis: Case report and literature review. Curr Oncol 19: e36-e41, 2012.

15. Wanless IR: Micronodular transformation (nodular regenerative hyperplasia) of the liver: A report of 64 cases among 2,500 autopsies and a new classification of benign hepatocellular nodules. Hepatology 11: 787-797, 1990.

16. Ghabril $M$ and Vuppalanchi R: Drug-induced nodular regenerative hyperplasia. Semin Liver Dis 34: 240-245, 2014.

17. Viganò L, Rubbia-Brandt L, De Rosa G, Majno P, Langella S, Toso C, Mentha G and Capussotti L: Nodular Regenerative Hyperplasia in Patients Undergoing Liver Resection for Colorectal Metastases After Chemotherapy: Risk Factors, Preoperative Assessment and Clinical Impact. Ann Surg Oncol 22: 4149-4157, 2015. 